\title{
Hydatid cyst-our institutional experience in hill area of Uttarakhand
}

\author{
Singh K. $\mathbf{P}^{1}$, Keshri $\mathrm{A}^{2}$, Prakash $\mathbf{S}^{3}$ \\ ${ }^{1}$ Dr Khem Pal Singh, Associate Professor, ${ }^{2}$ Dr Amit Keshri, Assistant Professor, ${ }^{3}$ Dr Sanjeev Prakash, Senior Resident, \\ all authors are affiliated with Department of General Surgery, HNB Base Hospital \& VCSGGMS \& RI, Srikot, Srinagar, \\ Pauri Garhwal, UT, India.
}

Address for Correspondence: Dr. Keshri Amit, Assistant Professor, Department of General Surgery, HNB Base Hospital \& VCSGGMS \& RI, Srikot, Srinagar, Pauri Garhwal, UT, India. E-mail : dramitkeshri@gmail.com

\begin{abstract}
Introduction: Hydatidosis caused by Echinococcus granulosus is a neglected zoonotic disease, widely prevalent among the rural population of India. Hepatic involvement is the commonest, with other varied presentations as well. Appropriate treatment consists of surgical excision of the cyst, accompanied with chemotherapy by albendazole or PAIR procedure. Methods: This retrospective analytic study was conducted to highlight and summarise the demography, modes of presentation and surgical management and outcomes of echinococcosis at VCSGGMS \& RI from years 2008-2016. Results: Most cases were adult male patients presenting with chronic manifestations of hydatidosis and/or abdominal lump (large hepatic cysts), caused by E. granulosus, who underwent surgical management (cyst excision), along with anthelmintics and later were followed up for appropriate durations, with only minor complications. Conclusion: Timely diagnosis and intervention can prevent morbidity, mortality and economic loss among the productive sections of the society.
\end{abstract}

Keywords: Hydatid Cyst, Echinococcus granulosus, Cystectomy, PAIR, Albendazole

\section{Introduction}

Hydatidosis [1-6] caused by echinococcus sp. tapeworm is widely prevalent in rural India, with varying presentations depending upon the organ of involvement, size of the cystic lesions and complications thereof $[2$, 9-13]. Contact with infected dog / sheep can result in disease; if recognised timely, medical \& or surgical traetment is readily available, to lessen its adverse impact upon the economically productive section of the population.

\begin{abstract}
Aim
In this study, we undertake to outline the epidemiology, presentations and outcome among the surgically managed patients of echinococcosis at our tertiary health care facility.
\end{abstract}

VCSGGMS \& RI and Base Hospital, Srikot, in hill region of Uttarakhand.

\footnotetext{
Manuscript received $4^{\text {th }}$ October 2016

Reviewed: $14^{\text {th }}$ October 2016

Author Corrected: $26^{\text {th }}$ October 2016

Accepted for Publication $10^{\text {th }}$ November 2016
}

\section{Material \& Methods}

In the backdrop of the above knowledge, we would like to present this retrospective observational study on the surgical management and outcome of patients of hydatidosis, mostly from hill areas of Uttarakhand, based upon our institutional experience at Base Hospital, VCSGGMS \& RI, Srikot, Uttarakhand, from June 2008 to August 2016. Data was collected, by review of case files, of all the patients who underwent surgical management of their condition and subsequently were confirmedly diagnosed with echinococcosis with HPE of the removed specimen / cyst, and analysis done, regarding cyst location, mode of surgical management, complications and outcome; also, follow-up analysis at appropriate intervals done in respect of recovery/recurrence / relapse of disease / symptoms etc. All the included patients had either presented to the Surgery OPD/ Emergency, or referred / transferred from Department of Medicine /Gynecology, and subsequently managed surgically under Department of Surgery. 
Exclusion/Inclusion Criteria: Patients having undergone only medical mode of management (after diagnosis by appropriate imaging/serology study) were not included in this study, albeit they outnumbered the surgically managed patients; some of those patients were discovered on imaging studies to even have multiple small hepatic cysts, causing diagnostic difficulties; many of such patients were managed as out-patients on albendazole chemotherapy and other supportive management for suitable duration, whence they showed radiological/general improvement, while few patients needed admission and close monitoring and/or prolonged chemotherapy for recovery.

All the above included patients underwent open surgical procedures (cyst excision/pericystectomy) (vs. laparoscopic or other means) after undergoing variable periods of pre-surgery albendazole chemotherapy; a right subcostal incision (not classic Kocher's incision) being the most frequently employed, with appropriate extensions (lateral/midline, vertical/left sub-costal extension) as required; otherwise, a midline abdominal incision was utilised. Omentopexy \& /or suture-closure of the leftover hepatic cavity was attempted in all cases, with a subhepatic drain insertion. In the cases with pulmonary involvement, formal lateral thoracotomies were done (a two-stage procedure in the patient with "liver + lung" involvement). Postoperative albendazole chemotherapy was administered to all; prolonged therapy resorted to in case of multi-site/ multiple/ extrahepatic cysts. Suitable antibiotics, blood transfusions and other supportive measures were used as warranted.

During the operative procedure, some of the patients with large sized/probably viable cysts were subjected to "AIR technique" (Photo \#6), whereby, after giving the laparotomy incision, the fluid cyst contents were aspirated, followed by instillation of scolicidal agents (20\% hypertonic saline)[25] and then further aspiration of the contents, repeatedly, before the the definitive surgical intervention, i.e., excision.

(Follow Up) All patients were followed-up for appropriate durations with review visits in the Surgery OPD and subsequent serial USG abdomen / X-ray chest / CT scan study.

\section{Results \& Observations}

As depicted in Table 1, male patients outnumbered the females, in contrast to various other studies. The peak incidence was in the socio-economically productive age-group (21 to $50 \mathrm{yrs}$ age) for both the sexes, while the next most-common group consisted of elderly patients. All patients came from rural/semi-urban background of Uttarakhand, from low socioeconomic strata, and all were following animal husbandry/agriculture practices/keeping pet dogs, exposing them in one way or another to the source of infection via the definitive/intermediate host of the Echinococcus; indeed, intimate contact with $\mathrm{dog} / \mathrm{sheep} / \mathrm{cattle}$ could be elicited in almost all cases.

Table -1: Demographic profile of patients included in study.

\begin{tabular}{|c|c|c|c|c|c|c|c|c|c|}
\hline \multirow{2}{*}{$\begin{array}{l}\text { Year } \\
20 x x\end{array}$} & \multicolumn{4}{|c|}{ Males } & \multicolumn{4}{|c|}{ Females } & \multirow{2}{*}{$\begin{array}{c}\text { Both } \\
(\mathrm{TM}+ \\
\text { TF })\end{array}$} \\
\hline & $<20 \mathrm{yrs}$ & $\begin{array}{c}21-50 \\
\text { yrs }\end{array}$ & $>50 \mathrm{yrs}$ & $\begin{array}{l}\text { Total } \\
\text { (TM) }\end{array}$ & $<20$ yrs & $\begin{array}{c}21-50 \\
\text { yrs }\end{array}$ & $>50 \mathrm{yrs}$ & $\begin{array}{l}\text { Total } \\
\text { (TF) }\end{array}$ & \\
\hline 68 & 0 & 1 & 1 & 2 & 0 & 0 & 0 & 0 & 2 \\
\hline 69 & 0 & 2 & 0 & 2 & 0 & 1 & 1 & 2 & 4 \\
\hline ‘ 10 & 0 & 2 & 1 & 3 & 0 & 0 & 1 & 1 & 4 \\
\hline '11 & 1 & 0 & 2 & 3 & 1 & 1 & 1 & 3 & 6 \\
\hline$\cdot 12$ & 0 & 2 & 1 & 3 & 0 & 2 & 0 & 2 & 5 \\
\hline$' 13$ & 0 & 1 & 1 & 2 & 0 & 2 & 0 & 2 & 4 \\
\hline '14 & 1 & 2 & 0 & 3 & 1 & 1 & 1 & 3 & 6 \\
\hline$' 15$ & 0 & 3 & 2 & 5 & 1 & 1 & 2 & 4 & 9 \\
\hline 16 & 1 & 1 & 1 & 3 & 0 & 1 & 1 & 2 & 5 \\
\hline \multirow{2}{*}{ Overall } & 3 & 14 & 9 & 26 & 3 & 9 & 7 & 19 & \multirow{2}{*}{45} \\
\hline & \multicolumn{4}{|c|}{26} & \multicolumn{4}{|c|}{19} & \\
\hline
\end{tabular}


Most of the patients complained of lump/pain in abdomen during time of admission, and hepatic involvement (single organ, right lobe) was the most common site of presentation, with occasional extra-hepatic manifestation as well (Table2). Multiple cysts were present in seven of the forty-five cases, most of them hepatic; two of them had bilobar hepatic involvement; all of them were excised in the same sitting (Table -3). Most patients had large size cysts at the time of admission/operative intervention.

Table-2: Site wise distribution of cyst lesions in patients included in study (Also, Photo \#1 - \#5).

\begin{tabular}{|c|c|c|c|}
\hline Site & Males (26) & Females (19) & Both (45) \\
\hline Liver & 23 & 17 & 40 \\
\hline Kidney & 1 & 0 & 1 \\
\hline Lung & 1 & 0 & 1 \\
\hline Pelvis/Sacral & 1 & 0 & 1 \\
\hline Liver + Spleen & 0 & 1 & 1 \\
\hline Liver + Lung & 0 & 1 & \\
\hline
\end{tabular}

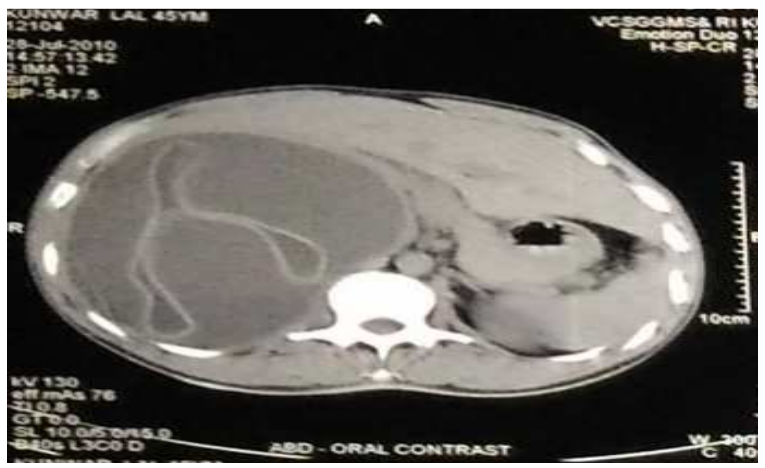

Figure 1: Hepatic hydatid cyst (right lobe unicystic) with "undulating membrane" inside.

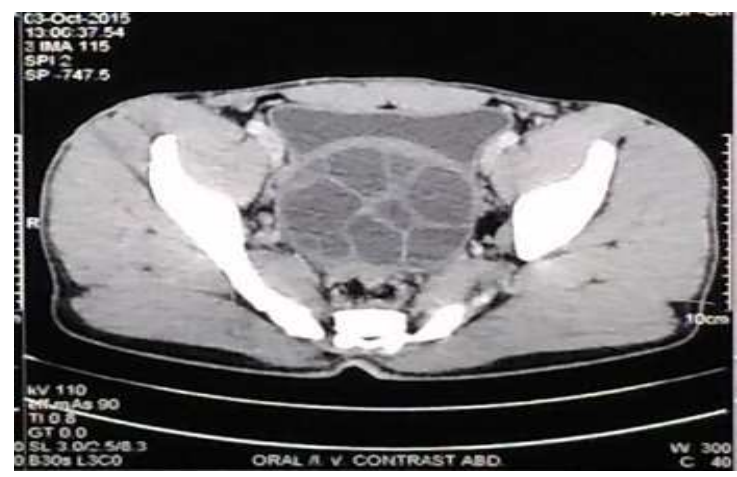

Figure 2: Pelvic/sacral hydatid cyst with multiple daughter cysts inside.
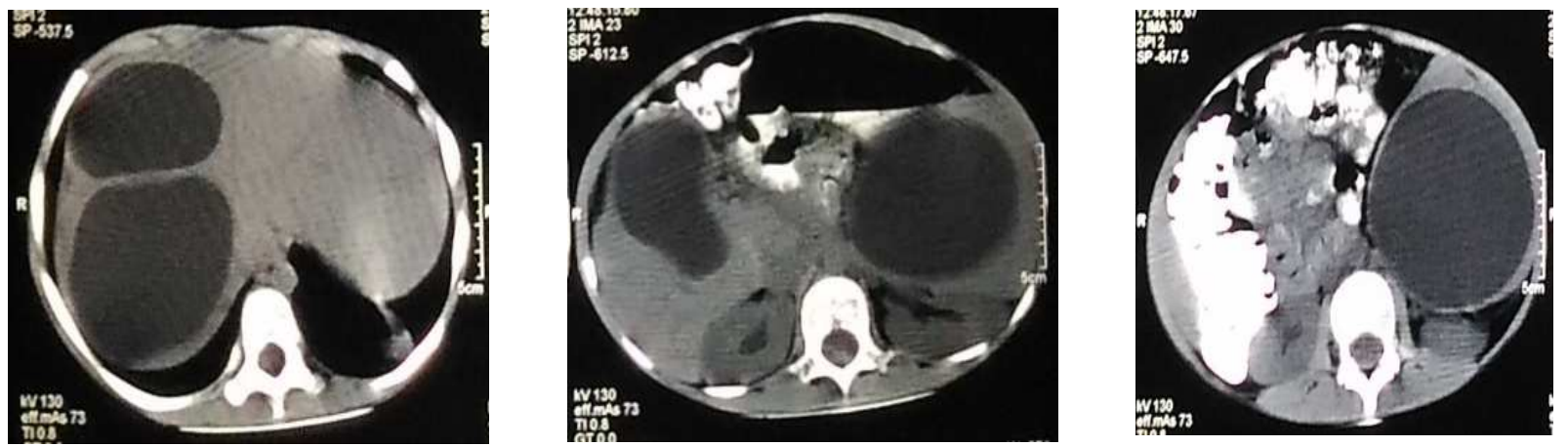

Figure 3 (a,b,c): Simultaneous hepatic (Multicystic) and splenic hydatid involvement. 


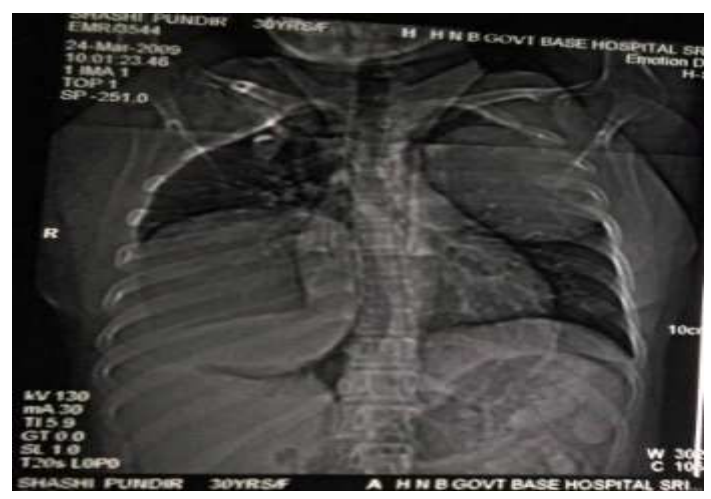

Figure 4: Simultaneous hepatic and pulmonary (Left) hydatid involvement.

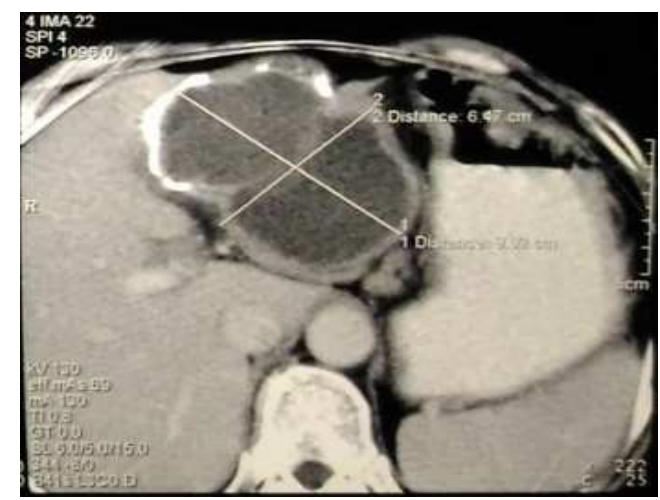

Figure 5: Multi-vesicular Hydatid Cyst in Left Hepatic Lobe with Partial Wall Calcification.

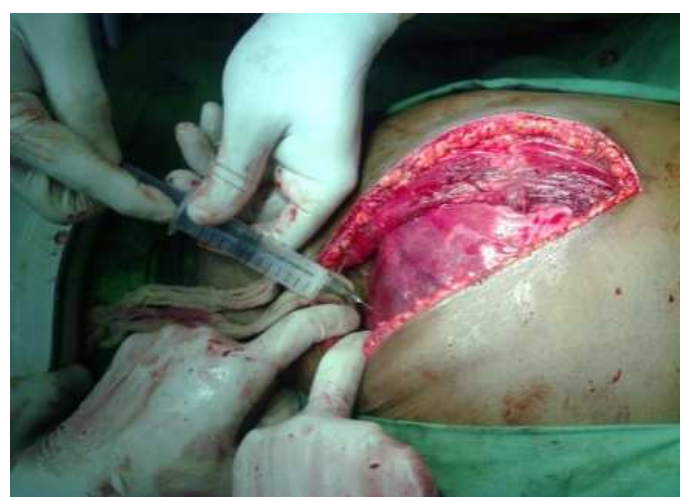

Figure 6: "AIR" Technique

Table-3: Cyst Characteristics. (All cysts were of E. granulosus on HPE study) (Photo \#1 - \#5).

\begin{tabular}{|c|c|c|c|c|c|}
\hline \multirow{2}{*}{ Site } & \multirow{2}{*}{$\begin{array}{l}\text { Cyst } \\
\text { Characteristics / } \\
\text { Comment }\end{array}$} & \multicolumn{2}{|c|}{ Cyst Number } & \multicolumn{2}{|c|}{ Cyst Size (diameter, cm) : Intra-Op } \\
\hline & & Single & Multiple & $<5 \mathrm{~cm}$ & $=>5 \mathrm{~cm}$ \\
\hline \multirow{3}{*}{ Liver (40) } & Males (23) & 20 & 3 & 5 & 18 \\
\hline & Females (17) & 14 & 3 & 3 & 14 \\
\hline & \multicolumn{5}{|c|}{$\begin{array}{l}\text { Five of the forty cases had multiple daughter cysts inside the main cyst; } \\
\text { Two of the six cases of multiple cysts had involvement of both lobes; } \\
\text { Otherwise, rest all of the hepatic cysts were in the right hemi-liver }\end{array}$} \\
\hline Kidney (1) & \multicolumn{5}{|c|}{ Single cyst, $\approx 10 \mathrm{~cm}$ diameter, with daughter cysts inside } \\
\hline Lung (1) & \multicolumn{5}{|c|}{ Single cyst in right lung, $\approx 15 \mathrm{~cm}$ diameter, no daughter cysts } \\
\hline Pelvis/Sacral (1) & \multicolumn{5}{|c|}{ Single cyst, $\approx 15 \mathrm{~cm}$ diameter, with daughter cysts inside } \\
\hline Liver + Spleen (1) & \multicolumn{5}{|c|}{ Multiple cysts in liver, solitary in spleen; all $>10 \mathrm{~cm}$ diameter; none with daughter cysts } \\
\hline Liver + Lung (1) & \multicolumn{5}{|c|}{ Solitary liver and solitary left lung cyst, both $>12-15 \mathrm{~cm}$ diameter; no daughter cysts } \\
\hline
\end{tabular}

All the cases included here were caused by E. granulosus infection, as confirmed by HPE study of the excised specimen (viable/dead cyst + part of parent tissue); infection with the other Echinococcus species could not be documented. 
No deaths or severe adverse reactions, including anaphylactic shock, occurred in any of the patients peri/post operatively, or during follow-up, though some patients had minor wound complications, including minor bile/blood leak in the initial few postoperative days (in three of the cases with hepatic cyst excision, minor bile leak in the intra-abdominal drain persisted for 15-20 days; resulting in delayed recovery, but otherwise uneventful, not requiring any further intervention), and/or delayed wound healing, and sometimes prolonged convalescence, especially in elderly. No spill out of cystic fluid or inadvertent rupture occurred during the surgeries or perioperatively, partly due to the preoperative course of albendazole (to kill/shrink the cysts), and also due to employment of meticulous intraoperative techniques (packing the operative site with pads soaked in $20 \%$ hypertonic saline / $10 \%$ Savlon scolicidal solution).

All patients were followed-up for reasonable periods of time following the operative intervention (up to six months to one year), depending upon duration of adjuvant albendazole therapy, resolution of symptoms and none of those patients showed any relapse/recurrence in the short-term follow-up period. Also interestingly, none of the above patients' household members/relatives/close contacts presented with the same disease.

\section{Discussion}

Hydatid disease (Hydatidosis / Echinococcosis) [1-6] is an important emerging neglected zoonotic disease of man, caused by the metacestode stage (infective larva) of the Echinococcus tapeworm [7,8]. Endemic in India $(\approx 1-200 / 105$ persons infected annually)[9], regions showing high prevalence are Andhra Pradesh (including Telangana), Tamil Nadu, Central India [10,11] and where animal husbandry is prevalent; though it is under-reported from the hilly northern states ( $\mathrm{J} \& \mathrm{~K}$, Uttarakhand, Himachal Pradesh) [12,13]. Those of major public health / clinical / economic importance in India are [2]:-

(a) Cystic Hydatid disease (CE)[14] $(\approx 95 \%$; various strains) (caused by E. granulosus complex, prevalent in livestock raising areas) - produces unilocular cystic lesions. The definitive hosts are canines; sheep, cattle, etc. act as intermediate hosts; and humans (rarely as intermediate), sometimes are "accidental dead-end hosts".

(b) Alveolar echinococcosis $(\mathrm{AE})[15](\approx 5 \%)$ (caused by E. multilocularis) produces multilocular alveolar locally-invasive cystic lesions. Canines, sp. foxes are the definitive and rodents the intermediate hosts.

(c) Rarely $[16,17]$ (probably absent in India), E. vogeli (polycystic echinococcosis), E. oligarthrus (unicystic), and other subtypes - E. shiquicus, E. Felidis.

After accidental ingestion of echinococcal eggs by humans, embryos penetrate the intestinal mucosa, enter the portal circulation and are carried to the liver, lungs and other organs [18-20]. Larvae develop into fluidfilled hydatid cysts (unilocular - E. granulosus; multilocular - E. multilocularis), that consist of an external membrane and an inner germinal layer.
Daughter cysts and germinating cystic structures (brood capsules) develop from the inner aspect of germinal layer. Protoscolices (new larvae) develop within the brood capsule. The cysts expand slowly over a period of years [1-6].

Slowly enlarging echinococcal cysts become symptomatic when their expanding size or their spaceoccupying effect in an involved organ elicits symptoms; they are sometimes discovered incidentally. Hepatic echinococcosis patients may present with abdominal pain or a palpable mass in the right upper quadrant; biliary obstruction can result in jaundice. Rupture of or episodic leakage from a hydatid cyst may produce fever, eosinophilia, or anaphylaxis. Pulmonary hydatid cysts may rupture into the bronchial tree or peritoneal cavity and produce cough, dyspnea, or hemoptysis[1-6].

Rupture of hydatid cysts (hepatic, etc.), (spontaneously / minor trauma or at surgery), may lead to multifocal dissemination of protoscolices, which can form additional cysts. Other presentations [1-6,21-23] are due to the involvement of extrahepatic organs, e.g., spleen, omentum, bone (invasion of the medullary cavity with slow bone erosion producing pathologic fractures), the CNS (space-occupying lesions), the heart (conduction defects, pericarditis), and the pelvis (pelvic mass).

The larval forms of E. multilocularis characteristically present as a slowly growing hepatic tumor, with progressive destruction of the liver and extension into adjoining structures (e.g., diaphragm, kidneys, or lungs) or may metastasize to the spleen, lungs, or brain[1-6,2123]. Radiographic and related imaging studies (plain $X$ rays, USG, CT/MRI) are important in detecting and evaluating/staging echinococcal cysts and classifying 
them [1-6]. The most pathognomonic finding, if demonstrable, is that of daughter cysts within the larger cyst. A specific diagnosis can also be made by examining aspirated fluids for protoscolices or hooklets, but there is risk of fluid leakage resulting in either dissemination of infection or anaphylactic reactions. Serodiagnostic assays can be useful (hepatic cysts elicit positive antibody responses in $\approx 90 \%$ of cases, whereas $\approx 50 \%$ of individuals with pulmonary cysts are seronegative). Detection of antibody to specific echinococcal antigens by immunoblotting has the highest degree of specificity [21].

Therapy [1-6] for cystic echinococcosis is based on considerations of the size, location, and manifestations of cysts and overall health of the patient. Surgery has traditionally been the principal definitive method of treatment. PAIR (percutaneous aspiration, infusion of scolicidal agents, and re-aspiration) is also a successful technique; contraindicated for superficially located cysts (because of the risk of rupture), for cysts with multiple thick internal septal divisions (honeycombing pattern), and for cysts communicating with the biliary tree. Concurrent/adjunctive albendazole chemotherapy is given with either procedure; albendazole alone may suffice for small, uncomplicated lesions[1-6].

Surgery (cystectomy, pericystectomy)[1-6] is the treatment of choice for complicated E. granulosus cysts or for areas where PAIR is not possible. Response to treatment is assessed by serial imaging studies; some cysts may not demonstrate complete radiologic resolution even though no viable protoscolices are present - managed with observation only. Complete surgical resection is the treatment of choice for $E$. multilocularis infection, accompanied with indefinite treatment with albendazole[1-6,24].

Dog/sheep vaccination[26,27], proper disposal of carcass of infected animal, getting rid of stray dogs, maintaining proper sanitation and thorough cooking of food before consumption are some means that can help reduce incidence of Echinococcosis [28].

\section{Conclusion}

Echinococcosis is prevalent in India with higher incidence among rural populace, especially in those who come in contact with dog/sheep/cattle, either while pursuing their means of livelihood or keeping such animals as pets. Echinococcus granulosus infection is being reported from most states of India now, and public education regarding means of spread of this preventable but neglected zoonotic disease and modes of prevention is the need of the hour. The economic burden could be further reduced by diagnosing and instituting appropriate-for-stage medical / surgical treatment at the earliest. Besides USG / CT of the abdomen (or imaging of other involved organ) and some highly specific serologic tests for its diagnosis, chemotherapy (with albendazole, etc.) is readily available at health centres (both government / private, small / large setups), across the country. PAIR procedure may be undertaken by the Surgeon /Interventional Radiologist / Physician, in suitable cases, at centres with facilities. Also, there is no dearth of many skilled and experienced surgeons (General / GI / Thoracic) throughout the length and breadth of the country who can manage and treat such cases efficiently and effectively, both by open and laparoscopic techniques.
Abbreviations

$\begin{array}{ll}\text { AE } & - \text { Alveolar Echinococcosis } \\ \text { CE } & - \text { Cystic Echinococcosis } \\ \text { CNS } & \text { - Central Nervous System } \\ \text { CT } & - \text { Computed Tomography } \\ \text { E. sp } & - \text { Echinococcus sp. } \\ \text { HPE } & - \text { HistoPathology Examination } \\ \text { MRI } & - \text { Magnetic Resonance Imaging } \\ \text { OPD } & - \text { OutPatient Department } \\ \text { PAIR } & - \text { Percutaneous Aspiration, Infusion } \\ & \text { and Re-aspiration } \\ \text { USG } & - \text { Ultrasonogram / Ultrasonography }\end{array}$

VCSGGMS\&RI - Veer Chandra Singh Garhwali Government Medical Sciences \& Research Institute

\section{Author Disclosure Statement}

Source of support : nil

Conflict of interest : none

Appropriate Ethical Clearance sought from Institutional Ethical Committee.

Funding: Nil, Conflict of interest: None initiated, Permission from IRB: Yes

\section{References}

1. Krige J, Bornman PC, Belghiti J. Hydatid Disease of Liver. Blumgart's Surgery of the Liver, Biliary Tract and Pancreas. 5ed. p1035-51.

2. Park K. Hydatid Disease. Park's Textbook of Preventive And Social Medicine. 21ed. p303-4. 
3.Zinner MJ, Ashley SW. Maingot's Abdominal Operations. Christians KK, Pitt HA. Hepatic Abscess And Cystic Disease Of The Liver. 12ed. p914-20.

4. Harrison's Principles of Internal Medicine. 19ed. Chap-260. p1432-34.

5. World Health Organization. Echinococcosis. www. who. int/ echinococcosis/about_disease/en/

6. Wikipedia Echinococcosis. https://en.wikipedia. org/ wiki/Echinococcosis

7. da Silva AM. Human Echinococcosis: A Neglected Disease. Gastroenterology Research and Practice Volume 2010, Article ID 583297, 9 pages. doi:10.1155/ 2010/583297

8. Singh BB, et al. Human hydatidosis: an under discussed occupational zoonosis in India. Helminthologia, 50, 2: 87 - 90, 2013. DOI: 10.2478/s 11687-013-0113-7.

9. Sharma M, Sehgal R, Fomda BA, Malhotra A, Malla N. Molecular characterization of Echinococcus granulosus cysts in north Indian patients: identification of G1, G3, G5 and G6 genotypes. PLoS Negl Trop Dis. 2013 Jun 13;7(6):e2262. doi: 10.1371/journal.pntd. 0002262 . Print 2013.

10. Rao SS, Mehra B, Narang R. The spectrum of hydatid disease in rural central India: An 11-year experience.Ann Trop Med Public Health 2012;5:225230. DOI: $10.4103 / 1755-6783.98624$.

11. Md Khader Faheem $\mathrm{N}$ et al. Scenario of Hydatid cyst disease in epidemic areas of Andhra Pradesh evaluation and analysis. Int J Res Dev Health. August 2013; Vol 1(3): $120-8$.

12. Fomda BA, Khan A, Thokar MA, Malik AA, Fazili A, Dar RA, Sharma M, Malla N. Sero-epidemiological survey of human cystic echinococcosis in Kashmir, North India. PLoS One. 2015 Apr 27;10(4):e0124813. doi: 10.1371/ journal. pone. 0124813. eCollection 2015.

13. Alexander PV, Rajkumar D. The pattern of hydatid disease-a retrospective study from himachal pradesh, India. Indian J Surg. 2010 Aug;72(4):331-5. doi: 10. 1007/s12262-010-0120-6. Epub 2010 Nov 23.
14. Anand S, Rajagopalan S, Mohan R. Management of liver hydatid cysts. Current perspectives. Medical Journal Armed Forces India 68 (2012) 304 e309. doi:10.1016/j.mjafi.2012.04.010.

15. Torgerson PR, Keller K, Magnotta M, Ragland N. The global burden of alveolar echinococcosis. PLoS Negl Trop Dis. 2010 Jun 22;4(6):e722. doi: 10.1371/ journal.pntd.0000722.

16. D'Alessandro A, Rausch RL. New Aspects of Neotropical Polycystic (Echinococcus vogeli) and Unicystic (Echinococcus oligarthrus) Echinococcosis. Clinical Microbiology Reviews. 2008;21(2):380-401. doi: 10.1128/CMR.00050-07

17. Adel Spotin. Echinococcus shiquicus and Echinococcus felidis. http://cdn.intechopen.com/pdfswm/48554.pdf http://dx.doi.org/10.5772/60819.

18. Moro P, Schantz PM. Echinococcosis: a review. International Journal of Infectious Diseases (2009) 13, 125-133. doi:10.1016/j.ijid.2008.03.037

19. Kulkarni P, Kumar D S. Food-borne parasitic infestations in India: Need for attention towards unattended. Int J Health Allied Sci 2014;3:213-5. DOI: $10.4103 / 2278-344 X .143047$.

20. Sarkar M, Pathania R, Jhobta A, Thakur BR, Chopra R. Cystic pulmonary hydatidosis. Lung India. 2016 Mar-Apr;33(2):179-91. doi: 10.4103/0970-2113. 177449.

21. Giri S, Parija SC. A review on diagnostic and preventive aspects of cystic echinococcosis and human cysticercosis. Trop Parasitol. 2012 Jul;2(2):99-108. doi: 10.4103/2229-5070.105174.

22. Mishra Pramod K. Textbook of Surgical Gastroenterology. Chap-67 p766-781. ISBN 9789351529989

23. Abbey RK, Sharma RK, Parshad S, Sood PC. Hydatid Cysts at Multiple Unusual sites in a Patient. Indian J Surg 2002; 64:71-2.

24. Ihsan Alloubi (2013). Thoracic Hydatid Cyst: Clinical Presentation, Radiological Features and Surgical Treatment, Principles and Practice of Cardiothoracic Surgery, Dr. Michael Firstenberg (Ed.), 


\section{Original Research Article}

InTech, DOI: 10.5772/53533. http://www.intechopen. com/books/principles-and-practice-of-cardiothoracicsurgery/thoracic-hydatid-cyst-clinical-presentationradiological-features-and-surgical-treatment.

25. H. Besim, K. Karayalin, O. Hamamci, Gongor And A. Korkmaz. Scolicidal Agents in Hydatid Cyst Surgery. HPB Surgery, 1998, Vol. 10, pp. 347-351. http: //dx.doi.org/10.1155/1998/78170.

26. Wenbao Zhang, et al. Vaccination of Dogs against Echinococcus granulosus, the Cause of Cystic Hydatid
Disease in Humans. The Journal of Infectious Diseases 2006; 194:966-74.

27. Parikh F. Echinococcosis--cut to cure but what about control? J Assoc Physicians India. 2012 Jul; 60:9-10.

28. Kayal A and Hussain A. A Comprehensive Prospective Clinical Study of Hydatid Disease. ISRN Gastroenterology Volume 2014, Article ID 514757. http://dx.doi.org/10.1155/2014/514757.

\section{How to cite this article?}

Singh K. P, Keshri A, Prakash S. Hydatid cyst-our institutional experience in hill area of Uttarakhand. Int J Med Res Rev 2016;4(11):2024-2031.doi:10.17511/ijmrr. 2016.i11.22. 